\title{
Advancing per- and polyfluoroalkyl substances (PFAS) research: an overview of ATSDR and NCEH activities and recommendations
}

\author{
Rachel D. Rogers ${ }^{1} \cdot$ Christopher M. Reh ${ }^{1} \cdot$ Patrick Breysse $^{1,2}$
}

Received: 12 November 2020 / Revised: 24 February 2021 / Accepted: 12 March 2021 / Published online: 1 April 2021

This is a U.S. government work and not under copyright protection in the U.S.; foreign copyright protection may apply 2021

\begin{abstract}
The National Center for Environmental Health (NCEH), part of the Centers for Disease Control and Prevention (CDC), and the Agency for Toxic Substances and Disease Registry (ATSDR) support and conduct research advancing national, state, and local public health response to per- and polyfluoroalkyl substances (PFAS). PFAS are a group of manufactured chemicals used in industry and consumer products that persist in the environment. Given the growing evidence linking PFAS with adverse health effects in humans, NCEH and ATSDR developed a public health research framework to capture the broad range of PFAS research activities being conducted and supported by the agency to determine future research priorities and identify opportunities for interagency collaboration. The framework was conceptualized via a multidisciplinary visioning process designed to identify compelling questions and research activities that span five scientific domains: toxicology, exposure, human health, public health action, and cross-cutting priorities. This paper presents a framework, compelling questions and research activities to help NCEH and ATSDR advance scientific discovery in partnership with federal, state, and local stakeholders as part of a comprehensive public health response to PFAS contamination.
\end{abstract}

\section{Introduction}

The Centers for Disease Control and Prevention's (CDC) National Center for Environmental Health (NCEH) and the Agency for Toxic Substances and Disease Registry (ATSDR) have developed a strategic vision and priorities for public health research on per- and polyfluoroalkyl substances (PFAS). These priorities focus on the potential human health effects of PFAS exposure within the United States based on evidence from studies spanning multiple scientific domains, including toxicology, exposure, individual and population health. PFAS affect communities nationwide in various ways, and NCEH and ATSDR (herein referred to as NCEH/ATSDR or the agency) are uniquely positioned to generate research to support and protect communities from potential harm from exposure to

Rachel D. Rogers

idz7@cdc.gov

1 Agency for Toxic Substances and Disease Registry, Atlanta, Georgia

2 National Center for Environmental Health, Centers for Disease Control and Prevention, Atlanta, Georgia
PFAS. This paper presents an overview of NCEH/ATSDR's focus on PFAS as a significant environmental hazard, proposes a public health research framework for PFAS, and describes research priorities for PFAS aligned with the agency mission.

\section{PFAS overview}

PFAS are a class of chemicals characterized by a carbon chain that is either fully or partially fluorinated. The class includes more than 5000 chemicals that have been widely produced and used in industry and consumer products since the mid-twentieth century. PFAS toxicity and pharmacokinetics vary and are largely driven by chemical structure [1-3]. The most commonly studied PFAS are perfluorooctanoic acid (PFOA), perfluorooctane sulfonic acid (PFOS), perfluorohexane sulfonic acid (PFHxS), and perfluorononanoic acid (PFNA). PFAS are water-soluble, do not break down easily, and possess long serum half-lives (residence times) in humans [4]. PFAS have been used to make non-stick cookware, water-repellent clothing, stain-resistant fabrics and carpets, cosmetics, and solar panels. Various products that resist grease, water, and oil contain PFAS, including firefighting foams such as aqueous film-forming foam (AFFF). 


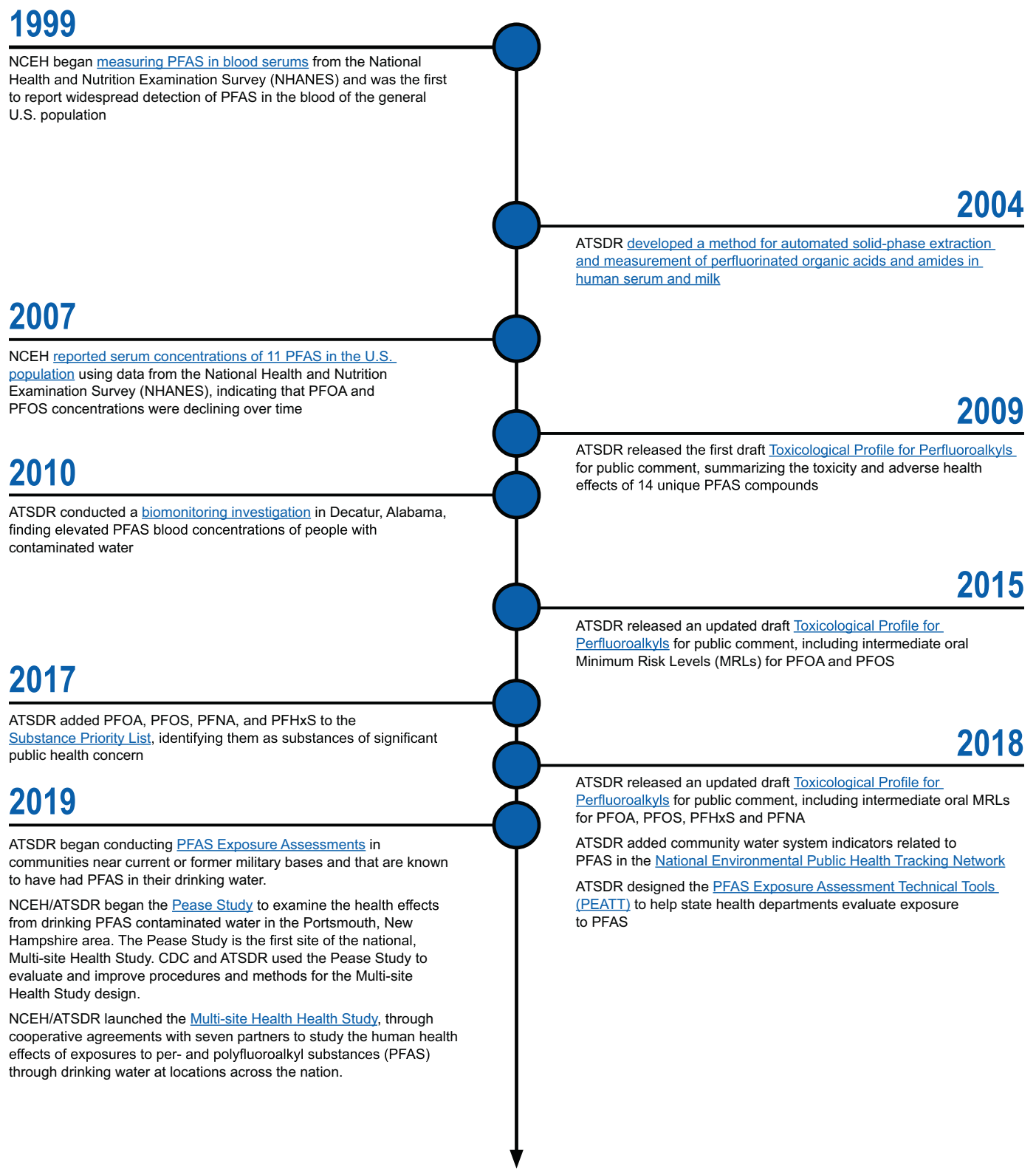

Fig. 1 Summary of NCEH/ATSDR PFAS Research Initiatives, 1999-2019. This timeline shows NCEH/ATSDR research activites over the past twenty years, demonstrating critical support for national, state, and local public health response to concerns about PFAS exposure.

Because of their unique properties, PFAS persist in the environment, and have been measured in people and wildlife worldwide [5].

A primary pathway of PFAS exposure in humans is drinking contaminated water near PFAS manufacturing and processing facilities, and near airfields, military installations, and fire-fighter training areas where AFFF was used [6]. As a result of federal action and growing public awareness of potential risks from widespread exposure to PFOA and PFOS, production and use of these two chemicals in the United States have declined since the early 2000s [7]. However, exposure to PFOS and PFOA still occur given their persistence in the environment. The majority of research on human health impact has focused on PFOS and PFOA, but as these compounds are phased out, people might be exposed to other PFAS. To date, PFAS have been detected in water systems, air, soil, and the food supply [7-9].

Research has linked PFAS exposure with adverse health effects in humans, but most studies have been conducted in laboratory animals. Findings from some epidemiological studies indicate exposure to certain PFAS increases the risks for some cancers. Exposure also might lead to increased cholesterol levels, changes in liver enzymes, decreased vaccine response in children, increased risk of high blood pressure or pre-eclampsia in pregnant women, and small 
decreases in infant birth weights $(<20 \mathrm{~g}$ or 0.7 ounce decrease in birth weight per $1 \mathrm{ng} / \mathrm{mL}$ increase in PFOA or PFOS in blood) [7].

Our understanding of the relationship between PFAS exposure and human health is incomplete due to limitations in study sample size, paucity of data for less-studied PFAS, over-reliance on mortality data, variation in research methodology, and co-exposure to mixtures of PFAS [7, 10]. Preliminary studies indicate newer PFAS might have properties similar to older PFAS chemicals, but more information is needed on chemical structures, behavior in the environment, and accumulation in the body from prolonged exposure. Addressing these questions will require targeted research and new analytical methods. It also will require federal inter-agency coordination and cross-sector partnerships to advance our understanding and ability to mitigate the human health effects of PFAS exposure.

\section{NCEH and ATSDR's history of advancing PFAS research for public health}

NCEH/ATSDR research activities have been instrumental in supporting national, state, and local public health response to PFAS for over 20 years (Fig. 1). In 1999, CDC added measures of 11 PFAS, perfluorooctane sulfonamide (PFOSA), 2-(N-ethyl-perfluorooctane sulfon-amido) acetic acid (Et-PFOSA-AcOH), 2-(N-methyl-perfluorooctane sulfonamido) acetic acid (Me-PFOSA-AcOH), perfluorobutane sulfonic acid (PFBuS), perfluorohexane sulfonic acid (PFHxS), PFOS, PFOA, perfluoroheptanoic acid (PFHpA), perfluorononanoic acid (PFNA), perfluorodecanoic acid (PFDeA), perfluoroundecanoic acid (PFUA), and perfluorododecanoic acid (PFDoA), to the National Health and Nutrition Examination Survey (NHANES). Through the analysis of NHANES data, NCEH's National Biomonitoring Program provides national reference values for PFAS exposure. The Toxicological Profile for Perfluoroalkyls (PFAs), first published in 2009 and continually updated with new scientific evidence, describes the toxicology and adverse health effects of select PFAS [7]. ATSDR has investigated PFAS exposure at more than 40 sites, either directly or through assisting state and federal partners [11]. The National Defense Authorization Act for Fiscal Year 2018 directs NCEH/ATSDR to conduct a range of activities to advance understanding of PFAS exposure and resulting potential threats to human health [12].

\section{Developing a public health research framework for PFAS}

NCEH/ATSDR has developed a comprehensive research framework to capture the broad range of PFAS-related research activities conducted and supported by NCEH/ ATSDR. To start, we identified five primary domains of PFAS research:

(I) Toxicology: Investigating the toxicological properties of PFAS to understand the adverse effects of PFAS exposure in living organisms.

(II) Exposure: Expanding knowledge of PFAS in the environment and exposure in individuals and populations.

(III) Human health: Examining the association between PFAS exposure in humans and individual, community, and population-level health outcomes.

(IV) Public health action: Developing and facilitating dissemination and implementation of a broad range of public health interventions and practices to prevent or reduce PFAS exposure or mitigate the risks of exposure to PFAS.

(V) Cross-cutting priorities: Addressing development of new or improved research methods across domains
Fig. 2 NCEH/ATSDR

Research Framework for

PFAS. This figure illustrates the interrelationships among scientific domains within the NCEH/ATSDR approach to PFAS concerns.

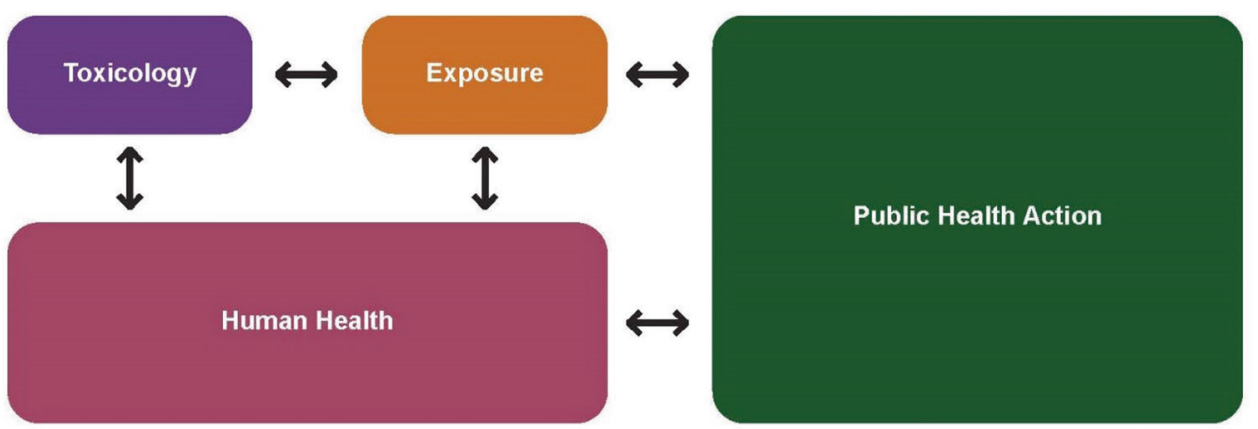

Cross - Cutting Priorities 
Table $1 \mathrm{NCEH/ATSDR} \mathrm{compelling} \mathrm{questions} \mathrm{for} \mathrm{PFAS} \mathrm{and} \mathrm{ongoing} \mathrm{role.}$

\begin{tabular}{ll}
\hline NCEH/ATSDR compelling questions for PFAS & NCEH/ATSDR ongoing role
\end{tabular}

1. Toxicology

1.1 What are the critical windows of exposure and the toxicokinetic (absorption, distribution, metabolism, excretion, clearance mechanisms, and biological halflife) and toxicodynamic (mechanisms of toxicological action) properties of individual PFAS, including less-studied and emerging types and common mixtures of PFAS?

1.2 How does chemical structure affect the physical, chemical, and biological behavior of PFAS?

1.3 How can PFAS be grouped according to their toxicological profiles?

2. Exposure

2.1 How can identification of individuals and communities exposed to PFAScontaminated drinking water be improved?

2.2 What factors affect the likelihood of PFAS exposure from non-drinking water sources?

2.3 What are the most appropriate biomarkers of exposure for PFAS, including less-studied and emerging species?

2.4 What are the most common mixtures of PFAS in drinking water? contaminated drinking water?

2.6 What are the PFAS biomonitoring concentration profiles associated with average and background exposure (not with a site-specific source, such as contaminated drinking water)?

2.7 What is the contribution of food to PFAS exposure?

2.8 What is the contribution of soil and dust to PFAS exposure?

2.9 What is the contribution of wastewater, bio-solids, and other recycled materials to PFAS exposure?

3. Human health

3.1 What is the association, if any, between PFAS exposure and the following health conditions? For each, how do observed associations differ for different PFAS mixtures?
- Ongoing revision and update of the agency's toxicological profiles for PFAS. - Determining which toxicological endpoints and mechanisms are most relevant to human health.

- Translating basic toxicology research into guidance values or minimum risk levels (MRLs) that are a critical tool for evaluating human PFAS exposure.

- Using computational methods, including physiologically based pharmacokinetic (PBPK) modeling and quantitative structure activity relationship (QSAR) modeling, to build the knowledge base for less well-characterized PFAS and to inform clustering or grouping PFAS for class-based evaluation.

- Developing an interactive geographic information systems (GIS) tool to help identify locations in the United States that have a higher risk for PFAS contamination, including identification and mapping of industries/location where PFAS are made or used.

- Enabling researchers and public health professionals to track PFAS exposure in drinking water through EPA's Unregulated Contaminant Monitoring Rule data in the NEPHTN.

- Conducting exposure assessments in communities with PFAS-contaminated drinking water.

- Supporting state, tribal, local, and territorial (STLT) health departments to conduct PFAS biomonitoring activities to characterize exposure in communities with contaminated drinking water through the PFAS exposure assessment technical tools (PEATT).

- Providing laboratory biomonitoring analytical support and research guidance to state, local, and academic PFAS studies.

- Collecting information from communities about behaviors that influence PFAS exposure from dietary sources to evaluate the contribution of the dietary pathway. - Assessing exposure to PFAS in environmental media in communities affected by contamination.

- Monitoring U.S. population exposure to PFAS using the National Health and Nutrition Examination Survey (NHANES) in the National Biomonitoring Program.

- Developing new methods for PFAS testing in the National Biomonitoring Program (e.g., urine).

- Conducting exposure assessments in communities with PFAS-contaminated drinking water to identify predictable exposure profiles.

- Developing state-of-the-art analytical methods for PFAS testing through the National Biomonitoring Program.

- Physiologically based pharmacokinetic modeling of human exposure to PFAS. - Developing models to historically reconstruct PFAS concentrations in communities.

- Monitoring groups of PFAS in the blood through NHANES

- Developing state-of-the-art analytical methods for PFAS testing through the National Biomonitoring Program.

- Identifying emerging needs for improved analytical detection methods for PFAS in environmental media other than water.

- Conducting a literature review to determine and evaluate existing data characterizing PFAS exposure from dietary sources.

- Collecting information from communities about behaviors that influence PFAS exposure from dietary sources to evaluate the contribution of the dietary pathway. - Supporting STLT health departments and other federal agencies in the identification and evaluation of dietary PFAS exposures (fish, game, cow's milk, etc.).

- Identifying emerging needs for improved analytical detection methods for PFAS in environmental media other than water.

- Assessing exposure to PFAS from dust samples in communities affected by PFAS contamination.

- Enhancing capacity of public health laboratories to expand access to PFAS testing in a variety of media.

- Identifying emerging needs for improved analytical detection methods for PFAS in environmental media other than water.

- Supporting efforts to build capacity in public health laboratories as they expand access to PFAS testing in a variety of media.

- Supporting risk assessments for exposures to PFAS through pathways other than drinking contaminated water.

- Conducting epidemiologic studies to clarify the association between PFAS exposure and health outcomes. 
Table 1 (continued)

\begin{tabular}{ll}
\hline NCEH/ATSDR compelling questions for PFAS & NCEH/ATSDR ongoing role \\
\hline $\begin{array}{l}\text { a. Birth outcomes or developmental effects } \\
\text { b. Renal function }\end{array}$ & $\begin{array}{l}\text { - Conducting literature reviews on PFAS exposure and human health effects } \\
\text { including immunologic/inflammatory markers in humans and cancer. }\end{array}$
\end{tabular}

c. Liver function

d. Vaccine efficacy

e. Susceptibility to particular infectious diseases

f. Cancer/malignancies

g. Cardiovascular disease

h. Immune-mediated diseases

3.2 What factors influence the risk for PFAS-related health outcomes in individuals and populations (i.e., genetic characteristics, age, race/ethnicity, socioeconomic status, stress, pharmaceuticals)?

- Conducting a literature review to understand the role that environmental contamination events and community resilience play in stress and related health outcomes, and related strategies to foster and support resilience to stress from PFAS contamination.

- Conducting a qualitative assessment with community leaders and public health practitioners to understand the experience of living in PFAS-affected communities.

3.3 How might PFAS exposure affect potential susceptibility and severity of viral • Supporting research to explore the intersection between PFAS exposure and illness such as COVID-19? susceptibility to viral infection such as COVID-19, including an assessment to determine the association between PFAS serum concentrations and COVID-19 infection severity and SARS-CoV-2 antibody titers.

4. Public health action

4.1 Who are the most relevant stakeholders in PFAS-affected communities for translation and implementation of prevention research?

- Supporting regional community engagement and assessment of community needs related to PFAS.

- Conducting social media analysis to better understand how the public searches for PFAS-related information, accesses web-based resources, and shares information.

- Engaging pediatric environmental health specialty units (PEHSUs) across the country to support medical professionals responding to PFAS exposure in affected communities.

- Engaging the research community to advance dissemination and implementation science for PFAS response.

4.2 What are the most effective strategies to engage stakeholders for advancing PFAS public health research and practice?

4.3 How can health education and communication strategies for communities exposed to PFAS be enhanced?

- Developing educational materials for healthcare providers to address the concerns of PFAS-exposed patients.

- Collaborating with professional medical organizations to share information, discuss emerging questions related to PFAS exposure, and assess the needs of the healthcare provider community.

- Sponsoring grand rounds in communities across the United States to raise awareness about PFAS among practitioners.

- Engaging PEHSUs across the country to support medical professionals responding to PFAS exposure in affected communities.

- Promoting community engagement and assessment of community needs related to PFAS concerns throughout the country.

- Supporting a community assistance panel for the community near the former Pease Air Force Base in Portsmouth, New Hampshire, to understand community health concerns, provide information on PFAS exposure and ongoing research efforts, and learn about how best engage the community in research activities.

4.4 What are the clinical practice recommendations for addressing PFAS exposure - Developing educational materials for healthcare providers to address the concerns in patients?

concerns of PFAS-exposed patients.

- Collaborating with professional medical organizations to provide clinical practice guidance, share information, discuss emerging questions related to PFAS exposure, and assess the needs of healthcare providers.

- Sponsoring grand rounds in communities across the United States to raise awareness about PFAS among practitioners.

- Engaging Pediatric Environmental Health Specialty Units (PEHSUs) across the country to support medical professionals responding to PFAS exposure in affected communities.

4.5 How can capacity in public health laboratories to analyze environmental, food, $\bullet$ Conducting needs assessments in collaboration with national organizations and biological samples for PFAS be expanded? identify opportunities for capacity building in public health laboratories. - Partnering with public health laboratories through the State Biomonitoring Cooperative Agreement to increase states' capability and capacity to conduct biomonitoring. developed to measure PFAS in water or different media?

5. Cross-cutting priorities

5.1 What are the broader effects of PFAS contamination (e.g., economic, social, • Conducting a literature review to understand the role that environmental behavioral)? contamination events and community resilience play in stress and related health outcomes, and related strategies to foster and support resilience to stress from PFAS contamination.

5.2 What is the relationship between environmental contamination of PFAS and $\bullet$ Conducting a qualitative assessment with community leaders and public health psychosocial stress among individuals, families, and communities? practitioners to understand the experience of living in PFAS-affected communities.

5.4 How can groundwater contaminant fate and transport modeling and plume characterization be improved?

- Conducting expert panels to develop an improved model for the historical reconstruction of PFAS exposure. 
Table 1 (continued)

5.5 How can biomonitoring data be enhanced with more detailed exposure data (e.g., questionnaires, drinking water, dietary data, etc.)?

5.6 How can laboratory methods keep pace with the rapidly expanding catalog of PFAS in commercial use?

5.7 How can advances in computational toxicology be harnessed to better understand the effects of exposure?

5.8 How can current risk assessment methodologies be improved to inform development of health-based guidelines for PFAS and mixtures?

5.9 How can data standardization, data sharing, and advanced analytics enhance the value of data for PFAS research?

$1-4$, and examining of broader effects (e.g., economic burden) and systems level effects of PFAS.

Figure 2 illustrates the interrelationships among the scientific domains - between basic and applied research in exposure, toxicology, and human health, and its translation, dissemination, and implementation into public health action. For instance, an exposure assessment revealing PFAS exposure in specific communities can inform a study on the potentially adverse human health effects. In turn, research on human health outcomes can inform communityspecific exposure assessments where potential risk for PFAS contamination is present by setting thresholds in environmental levels that would trigger public health response. NCEH/ATSDR is actively pursuing research in all five domains with a particular emphasis on human health and public health action, giving the agency's focus on population health and public health practice.

Once the research domains were identified, The NCEH/ ATSDR PFAS Community of Practice (CoP), which includes agency staff ranging from toxicologists, medical officers, epidemiologists, health scientists, and chemists to health policy, communications, community engagement, data science, and geographic information systems (GIS) experts, identified priority PFAS research topics within each PFAS research domain. In February of 2019, an initial visioning session was conducted with the CoP to consider scientific advances and gaps in knowledge that, if addressed by NCEH/ATSDR, could improve the federal response to PFAS contamination. The visioning process resulted in an extensive set of research priorities with the potential to provide significant near- and long-term benefit to the national public health response to PFAS. The agency subsequently convened a subset of participants from the $\mathrm{CoP}$ in two design session in March and April of 2019 to refine the
- Designing questionnaires for health and exposure studies to enhance assessment of exposure and complement biomonitoring data.

Conducting computational toxicology to integrate in silico methods with traditional approaches.

- Developing MRLs for individual PFAS.

Exploring the utility of electronic health records as a surveillance tool to inform the design of larger epidemiologic investigations.

- Developing an interactive geographic information systems tool to help identify locations in the United States that have a higher risk for PFAS contamination.

- Implementing innovative biomonitoring report back methods to provide personalized exposure information with context to aid in interpretation of results across all levels of scientific literacy.

- Establishing a centralized data repository to include PFAS data sets collected or generated by NCEH/ATSDR funded projects, and external PFAS-related environmental, biomonitoring, and health data that are available for public use, purchase, or sharing via data use agreements.

initial set of PFAS research priorities. The final set of compelling questions were presented to the $\mathrm{CoP}$ and NCEH/ATSDR leadership to assess and prioritize their alignment with the agency mission and strategic plan.

\section{Compelling questions to advance research on PFAS}

Table 1 identifies compelling questions to drive PFAS research and will serve as the foundation for a multi-faceted strategy for advancing PFAS science. Each question identifies a significant gap within the five domains of the PFAS research framework that can be addressed by targeted action and investment by NCEH/ATSDR. The following domainspecific summaries highlight the state of the science in PFAS and the Agency's current research activities relevant to the priority topic.

\section{Toxicology}

The agency's primary role in the toxicology domain is applied toxicology. NCEH/ATSDR relies on basic toxicologic research conducted by academic institutions and other federal agencies, such as the National Institute for Environmental Health Science and the National Toxicology Program, for evaluation and risk assessment of human exposure to PFAS.

In this domain, NCEH/ATSDR prioritizes basic toxicological research to define the toxicokinetic and toxicodynamic properties, across life stages, of previously under-studied PFAS in order to support the development of guidance values or minimum risk levels (MRLs). In particular, the agency recognizes the need to determine the toxicological endpoints and mechanisms of action of newly 
developed or newly identified PFAS, to predict the biological activity of under-studied PFAS, and to assess exposures to mixtures of PFAS [13].

As a result of the long history of PFAS use and the environmental persistence of these compounds, many people have experienced chronic (e.g., lifetime) PFAS exposure. As such, NCEH/ATSDR places emphasis on the need for studies that evaluate the impact of long duration exposures. Such studies would enable the agency to develop public health tools to address the kinds of exposures that are most likely to have occurred.

NCEH/ATSDR also recognizes that data on critical windows of exposure for PFAS toxicity as well as individual factors that influence clearance rates, such as genetic characteristics, age, race/ethnicity, socioeconomic status, stress, chronic disease, and pharmaceutical use are sparse. Basic toxicologic research could elucidate these questions, allowing the agency to better identify vulnerable populations.

Finally, NCEH/ATSDR recognizes that risk assessment approaches for evaluating PFAS mixtures are needed, and that data-driven understanding of the exposure profiles, pharmacokinetic properties, molecular mechanisms, and toxicological effects of PFAS-containing chemical mixtures is an important research priority $[14,15]$.

To address these research gaps, NCEH/ATSDR scientists are using computational methods (e.g., physiologically based pharmacokinetic (PBPK) and quantitative structure activity relationship (QSAR) modeling) to build the knowledge base for less well-characterized PFAS and to inform clustering or grouping PFAS for class-based evaluation.

\section{Exposure}

Exposure investigation is at the core of the NCEH/ATSDR mission [7]. As such, the agency has identified many compelling questions in this domain to guide our PFAS research priorities.

PFAS exposure is complicated by the numerous chemicals, sources, and routes of exposure. To date, much concern has focused on contamination of drinking water. However, other pathways of exposure (e.g., inhalation, dermal, and non-drinking water related ingestion) are generating increasing attention. Past exposure assessment has focused on environmental contamination resulting from the use of PFAS and PFAS-containing materials. With the reduction in use and import of PFAS, the focus must shift to exposure resulting from the disposal and destruction of PFAS and PFAS-containing materials. The agency recognizes the growing importance of examining the contribution of non-drinking water exposure to PFAS, given the significant progress made towards mitigating contaminated drinking water.
NCEH/ATSDR places great priority on reduction of PFAS exposure. As a first step, this requires the identification of communities where PFAS exposure is occurring. Ongoing and planned drinking water surveillance efforts occurring across the federal family are critical for identifying communities with PFAS-contaminated drinking water. However, additional water testing is needed in communities that fall outside existing surveillance efforts, such as those that have small water systems or private wells $[6,16]$.

Further, the agency recognizes the need to shift from drinking-water focused surveillance to a broader approach that acknowledges the possibility of significant nondrinking water exposure. Accumulating evidence suggests that people can be exposed to PFAS through inhalation; swallowing contaminated food, breastmilk, dust, or soil; or possibly routes such as skin contact [7, 8, 17-19]. The agency sees an important opportunity to proactively identify geographic indicators (e.g., proximity to certain industrial facilities) or individual factors (e.g., diet and consumer behavior) associated with increased risks for exposure in order to support a more complete assessment of PFAS exposure [16]. NCEH/ATSDR is also focused on expanding exposure assessments in communities with known PFAS contamination to include more robust environmental sampling (e.g., dust, soil, air).

NCEH/ATSDR continues to measure a suite of PFAS in blood samples collected through the NHANES program. The agency strives to ensure that the analytical approach captures the most important information to shed light on PFAS exposure in the general US population. Prioritization of research on PFAS biomonitoring, as well as biomarkers of PFAS exposure and effect both within and outside the agency will inform exposure and health assessments, and potentially identify interventions to reduce PFAS exposure [7].

\section{Human health}

NCEH/ATSDR plays a significant role in examining the human health effects of PFAS and is focused on taking a class-based approach to understand the effects of exposure to mixtures of PFAS.

Inconsistencies among individual studies suggest a need for additional research to clarify the adverse effects on human health [7]. These include immune effects, certain malignancies, cardiovascular disease, developmental and reproductive effects, and other organ system effects $[4,7,10,20]$. Given emerging evidence that PFAS may impact the immune system, more research is needed to identify how PFAS exposure may affect the severity of viral illness (including, but not limited to COVID-19) [21, 22]. In particular, studies examining the association between health 
outcomes and exposure to PFAS mixtures are needed. Prospective cohort studies of exposed communities will help delineate causal relationships between PFAS exposure and human health effects to inform clinical, public health surveillance, and response recommendations.

NCEH/ATSDR recognizes the need to improve the understanding of vulnerability and susceptibility to health risks associated with PFAS exposure [23]. Specifically, the agency sees an opportunity to improve the understanding of how genetic characteristics, race/ethnicity, socioeconomic status, stress, pre-existing conditions, and certain behavioral characteristics may influence risk for PFAS-related health outcomes.

To fill these gaps, NCEH/ATSDR is conducting epidemiologic studies and developing guidance on PFAS risk assessment for exposure to PFAS mixtures.

\section{Public health action}

The public health action domain aims to identify public health interventions and practices to reduce exposure, prevent potential adverse health effects, and inform public health professionals, healthcare providers, and the public about the potential risks of PFAS exposure.

As prevention research on PFAS exposure and PFASrelated health outcomes evolves, translation and implementation science can help identify more effective strategies for individual and population-level interventions. More research is needed to determine effective messages, channels, and strategies for audiences ranging from healthcare providers to persons and communities exposed to PFAS. For example, social media has been effective in spreading messages and awareness of environmental concerns to communities, and thus warrants further exploration as a communications channel [24]. Identifying and assessing stakeholder needs and engaging communities throughout the research process can help increase the effectiveness of public health communications and interventions.

Clinical intervention research can inform treatment of individuals in a clinical setting and support the development of a comprehensive public health response to PFAS. Clinical guidance and continuing education may improve the capacity of healthcare providers to address the concerns of patients and communities affected by PFAS exposure.

Exposure prevention research can guide steps to reduce PFAS exposures in contaminated communities. Identification of the most appropriate policy, regulatory, systems, and environmental change strategies can improve our ability to prevent future exposures to PFAS at the population level. Potential strategies include identifying PFAS that should no longer be used in consumer products or identifying uses of PFAS that are non-essential and appropriate for elimination. Identifying innovative methods of PFAS mitigation and cleanup for water systems and other environmental media will help reduce PFAS exposure in affected communities [16].

To address gaps in the public health action domain, NCEH/ATSDR is focused on the applying evidence-based biomonitoring report back methods to provide personalized exposure information with context to aid in interpretation of results across all levels of scientific literacy.

\section{Cross-cutting priorities}

The cross-cutting priorities domain includes research to develop new and improved scientific and analytical laboratory methods to advance research across all domains. This category also includes efforts to improve coordination and prioritization of research across federal agencies and academia and assessment of the broader societal effects of PFAS exposure (i.e., economic burden and community resilience), which can affect (or be affected by) multiple scientific domains.

The broader societal effects of PFAS exposure and contamination have not been well-studied. Research is needed to examine the effects of PFAS exposure on socioeconomic factors in communities exposed to PFAS [23]. The economic burden of PFAS exposure might worsen the adverse effects of PFAS in resource-constrained populations unable to implement mitigation strategies including water filtration or relocation. Additionally, psychosocial stress related to PFAS exposures can pose health risks independent from toxicological risks, and community resilience can serve as a protective factor. Understanding these potential determinants of health will inform the development of targeted intervention research and public health action.

Innovative technologies can help address growing concerns about PFAS exposure. These include tools to assess the routes by which PFAS enter and move through the environment, improved environmental and human sampling techniques, and new metrics to characterize PFAS exposure. For example, innovative assays that measure total fluorine content or precursor chemicals in environmental and biological samples need to be assessed for application over traditional tests for targeted PFAS [16]. The development of low-cost, rapid testing tools to measure PFAS in water, dust, or other environmental media will help identify and prioritize targets for environmental intervention. Capacity building activities to support public health laboratories will improve access to high quality and standardized testing for an expanded range of PFAS and specimen types (i.e., environmental, food, and biological). The results will advance our understanding of the scope of exposure and support community actions to protect health. Including citizen 
science and real-time data collection into these efforts might enhance study outcomes, accessibility of data, and awareness of findings among individuals and communities [25]. Integration of in vitro, in vivo, and in silico methods improve understanding of the breadth of PFAS toxicity, generate additional data to support PFAS risk assessment, and guide epidemiologic study design. Additional research is needed to improve translation and dissemination science and enhanced data sharing. This research would benefit a broad range of activities and inform exposure guidelines for PFAS and PFAS mixtures.

NCEH/ATSDR's primary role to address these gaps includes applying the toxicological profile framework to develop public health guidance via minimum risk levels for PFAS, as well as applying computational toxicology to integrate in silico methods with traditional approaches.

\section{Next steps for advancing PFAS research priorities at NCEH/ATSDR}

NCEH/ATSDR intends to build upon its broad range of ongoing PFAS research activities to accelerate the public health response to PFAS. NCEH/ATSDR recognizes the importance of continued collaboration and communication with federal, state, and community partners to support research and avoid duplication of efforts.

\section{Toxicology}

NCEH/ATSDR is uniquely positioned to translate emerging toxicological research into actionable public health strategies to evaluate PFAS exposures and prioritize the need for public health action where exposure is occurring. As additional toxicological research becomes available to clarify the potential public health concern of a broad range of PFAS, NCEH/ATSDR can expand existing surveillance systems, including NHANES and the National Environmental Public Health Tracking Network (NEPHTN), to more fully characterize exposures that might affect public health. These systems can also support development of risk assessment approaches and the use of class-based approaches to examine the potential additive effects of PFAS mixtures to human health. Improved surveillance and proactive toxicological research can help identify biomarkers of exposure for less-studied and emerging PFAS chemicals and help prevent future exposures.

\section{Exposure}

NCEH/ATSDR, in coordination with federal and community partners, has several opportunities to develop a comprehensive understanding of PFAS exposure across the US.
Improved groundwater contaminant fate and transport modeling and plume characterization for PFAS will inform historical reconstructions of exposure. To better characterize occupational exposure, NCEH/ATSDR can support the tracking and comparison of exposure levels in specific occupational groups (e.g., firefighters).

Finally, NCEH/ATSDR can expand the analytical approaches and sampling strategies applied to exposure investigation in communities with PFAS exposures in order to address gaps in knowledge about non-drinking water sources and background exposure to PFAS. Adding measurements of PFAS concentrations in air, water, food, soil, and dust to the NEPHTN can improve the understanding of background exposure and the relative contribution of these pathways to total PFAS exposure. Applying methods to evaluate exposures to PFAS through pathways other than drinking contaminated water can inform and improve future exposure and human health research and public health action to prevent or reduce the effects of exposure.

Using geographic information system methods to characterize the introduction of PFAS into the environment (e.g., biosolid application, air releases, use of PFAS-containing materials, etc.) can improve the ability to predict areas and populations at risk for contamination. Collaboration with partners to share and improve environmental PFAS data can help identify communities in need of exposure assessment.

\section{Human health}

Although NCEH/ATSDR is making significant strides to expand our understanding of the human health effects of PFAS, confirmatory studies will be needed to validate the results of ongoing studies. Well-designed longitudinal studies to examine PFAS exposure over time will further our understanding of trends in levels and composition of PFAS exposure. The results can be used to evaluate the effectiveness of interventions to reduce exposure. Planning additional studies to make use of existing data or biologic specimens presents another cost-effective approach to expand the knowledge base. NCEH/ATSDR understands that addressing cancer endpoints are a major concern for some community members living in areas affected by PFAS contamination. Conducting analyses using previously collected data might help clarify associations between PFAS exposure and cancer. As PFAS exposure patterns are better understood, additional participants for current and proposed research protocols will be identified, along with new health studies investigating PFAS mixtures. Given the significant, unprecedented threat of COVID-19, NCEH/ATSDR is expanding support for assessing the potential human health impacts of PFAS exposure on susceptibility and severity of illness related to COVID-19. 


\section{Public health action}

NCEH/ATSDR can further support PFAS-related public health action through partnership with national medical associations to create clear clinical practice recommendations for healthcare providers. That might include guidance on educating patients at risk for PFAS exposure and addressing concerns of exposed persons. The agency recognizes that healthcare providers are an important source of information for affected communities. NCEH/ATSDR is uniquely situated to provide outreach and education in support of those communities. The expansion of these efforts can aid effective translation and dissemination of emerging science and research around PFAS and public health.

To enhance health education and communication in communities affected by PFAS contamination, NCEH/ATSDR can develop and use innovative biomonitoring report-back methods, such as interactive web-based tools, to provide personalized risk profiles. Efforts to build capacity in public health laboratories can increase access to standardized and qualityassured PFAS testing in communities. Furthermore, NCEH/ ATSDR can expand its efforts in community engagement and assessment of community needs related to PFAS contamination throughout the country. NCEH/ATSDR will continue to use the best available evidence to advise communities on how to reduce exposures. Sustained research on environmental cleanup will improve our ability to prevent exposure around the US.

\section{Cross-cutting priorities}

NCEH/ATSDR can continue to support the evaluation and application of innovative research methods across multiple domains of PFAS research. Key ongoing initiatives, such as exposure assessments and a multi-site health study, will provide significant opportunities to develop and evaluate new research methods. The agency can assess the value of emerging laboratory methods (e.g., total organic fluorine, total oxidizable precursors, and non-targeted analyses) for identification of total PFAS exposure. Studies to guide the development and scaling of community-based stress resilience interventions will improve the public health community's ability to address the role that chronic stress plays in the development of health effects in communities with PFAS exposures, including accounting for the immediate and long-term risk represented by COVID-19 to individuals and communities. Potential uses of electronic health records as a surveillance tool might inform the design of large epidemiologic investigations.

\section{Conclusion}

Scientific discovery remains central to NCEH/ATSDR's core mission. The agency continues to address health challenges by investigating the relationship between environmental factors and health. NCEH/ATSDR is determined to address the compelling questions presented in this report in response to the growing concern over PFAS. The activities undertaken by NCEH/ATSDR are evidence-based solutions to real environmental health needs and pertinent public health problems across the country. The science of PFAS is evolving; this report presents an overview of key questions and considerations for NCEH/ATSDR to advance knowledge surrounding PFAS. This effort will require targeted investment in research capacity as NCEH/ATSDR continues to work with other agencies and stakeholders through cooperative agreements, grants, and contracts that advance the vision of the agency.

NCEH/ATSDR aims to support research and programmatic priorities that are dynamic and responsive to the evolving scientific landscape and emerging opportunities to address PFAS. The agency will evaluate past and ongoing PFAS initiatives to identify opportunities to improve the response to emerging environmental health issues more broadly, emphasizing a proactive, population-level approach to best protect public health. This ongoing, iterative process will require continued engagement of stakeholders within NCEH/ATSDR, across other federal agencies, with state and local partner organizations, and in communities affected by PFAS. As PFAS knowledge and science grow, NCEH/ATSDR can leverage its unique capacity, resources, and staff to develop guidance, build partnerships, and implement environmental health programs that protect and promote health. The sustained participation, creativity, and commitment of these stakeholders and NCEH/ATSDR will enable achievement of the research priorities identified in this document.

As NCEH/ATSDR advances toward implementing the research recommendations mentioned in this report, the commitment to continued engagement with key stakeholders and the community affected by PFAS contamination is critical to advance these research priorities.

\section{Disclaimer}

The findings and conclusions in this report are those of the authors and do not necessarily represent the official position of the Centers for Disease Control and Prevention and/or the Agency for Toxic Substances and Disease Registry.

Funding This report was by supported by funds from the Agency for Toxic Substances and Disease Registry, U.S. Department of Health and Human Services. 


\section{Compliance with ethical standards}

Conflict of interest The authors declare no competing interests.

Publisher's note Springer Nature remains neutral with regard to jurisdictional claims in published maps and institutional affiliations.

\section{References}

1. Kudo N, Kawashima Y. Toxicity and toxicokinetics of perfluorooctanoic acid in humans and animals. J Toxicol Sci. 2003;28:49-57. https://doi.org/10.2131/jts.28.49.

2. Taves DR. Evidence that there are two forms of fluoride in human serum. Nature. 1968;217:1050-1. https://doi.org/10.1038/ $2171050 \mathrm{~b} 0$.

3. Buck RC, Franklin J, Berger U, Conder JM, Cousins IT, de Voogt $\mathrm{P}$, et al. Perfluoroalkyl and polyfluoroalkyl substances in the environment: terminology, classification, and origins. Integr Environ Assess Manag. 2011;7:513-41. https://doi.org/10.1002/ ieam.258.

4. Wang Z, DeWitt JC, Higgins CP, Cousins IT. A never-ending story of per- and polyfluoroalkyl substances (PFASs)? Environ Sci Technol. 2017;51:2508-18. https://doi.org/10.1021/acs.est.6b04806.

5. Lau C, Anitole K, Hodes C, Lai D, Pfahles-Hutchens A, Seed J. Perfluoroalkyl acids: a review of monitoring and toxicological findings. Toxicol Sci. 2007;99:366-94. https://doi.org/10.1093/ toxsci/kfm 128 .

6. Hu XC, Andrews DQ, Lindstrom AB, Bruton TA, Schaider LA, Grandjean $\mathrm{P}$, et al. Detection of poly- and perfluoroalkyl substances (PFASs) in U.S. drinking water linked to industrial sites, military fire training areas, and wastewater treatment plants. Environ Sci Technol Lett. 2016;3:344-50. https://doi.org/10. 1021/acs.estlett.6b00260.

7. Agency for Toxic Substances and Disease Registry. Toxicological Profile for Perfluoroalkyls. (Draft for Public Comment). Atlanta, GA: Agency for Toxic Substances and Disease Registry; 2018.

8. Sunderland EM, Hu XC, Dassuncao C, Tokranov AK, Wagner CC, Allen JG. A review of the pathways of human exposure to poly- and perfluoroalkyl substances (PFASs) and present understanding of health effects. J Expo Sci Environ Epidemiol. 2019;29:131-47. https://doi.org/10.1038/s41370-018-0094-1.

9. Calafat AM, Wong L-Y, Kuklenyik Z, Reidy JA, Needham LL. Polyfluoroalkyl chemicals in the U.S. population: data from the National Health and Nutrition Examination Survey (NHANES) 2003-2004 and comparisons with NHANES 1999-2000. Environ Health Perspect. 2007;115:1596-602. https://doi.org/10.1289/ehp. 10598.

10. Steenland K, Fletcher T, Savitz DA. Epidemiologic evidence on the health effects of perfluorooctanoic acid (PFOA). Environ Health Perspect. 2010;118:1100-8. https://doi.org/10.1289/ehp.0901827.

11. Agency for Toxic Substances and Disease Registry. How is ATSDR involved investigating PFAS in the environment? 2019. https://www.atsdr.cdc.gov/pfas/atsdr_sites_involvement.html.

12. United States. Congress. House. Committee on Armed Services. National Defense Authorization Act For Fiscal Year 2018 : Report of the Committee on Armed Services, House of Representatives on H.R. 2810 Together with Dissenting Views (Including Cost Estimate of the Congressional Budget Office). Washington: U.S. Government Publishing Office; 2017.
13. Post GB, Gleason JA, Cooper KR. Key scientific issues in developing drinking water guidelines for perfluoroalkyl acids: contaminants of emerging concern. PLoS Biol. 2017;15:e2002855-e2002855. https://doi.org/10.1371/journal.pbio.2002855.

14. Hu XC, Dassuncao C, Zhang X, Grandjean P, Weihe P, Webster GM, et al. Can profiles of poly- and perfluoroalkyl substances (PFASs) in human serum provide information on major exposure sources? Environ Health. 2018;17:11 https://doi.org/10.1186/ s12940-018-0355-4.

15. Rappazzo KM, Coffman E, Hines EP. Exposure to perfluorinated alkyl substances and health outcomes in children: a systematic review of the epidemiologic literature. Int J Environ Res Public Health. 2017;14:691 https://doi.org/10.3390/ijerph14070691.

16. Guelfo JL, Marlow T, Klein DM, Savitz DA, Frickel S, Crimi M, et al. Evaluation and management strategies for per- and polyfluoroalkyl substances (PFASs) in drinking water aquifers: perspectives from impacted U.S. Northeast communities. Environ Health Perspect. 2018;126:65001 https://doi.org/10.1289/ EHP2727.

17. Cariou R, Veyrand B, Yamada A, Berrebi A, Zalko D, Durand S, et al. Perfluoroalkyl acid (PFAA) levels and profiles in breast milk, maternal and cord serum of French women and their newborns. Environ Int. 2015;84:71-81. https://doi.org/10.1016/j. envint.2015.07.014

18. Worley RR, Yang X, Fisher J. Physiologically based pharmacokinetic modeling of human exposure to perfluorooctanoic acid suggests historical non drinking-water exposures are important for predicting current serum concentrations. Toxicol Appl Pharmacol. 2017;330:9-21. https://doi.org/10.1016/j.taap.2017.07.001.

19. D'eon JC, Mabury SA. Is indirect exposure a significant contributor to the burden of perfluorinated acids observed in humans? Environ Sci Technol. 2011;45:7974-84. https://doi.org/10.1021/ es200171y.

20. Shankar A, Xiao J, Ducatman A. Perfluorooctanoic acid and cardiovascular disease in US adults. JAMA Intern Med. 2012;172:1397-403. https://doi.org/10.1001/archinternmed.2012. 3393.

21. Grandjean P, Heilmann C, Weihe P, Nielsen F, Mogensen UB, Timmermann A, et al. Estimated exposures to perfluorinated compounds in infancy predict attenuated vaccine antibody concentrations at age 5-years. J Immunotoxicol. 2017;14:188-95. https://doi.org/10.1080/1547691X.2017.1360968.

22. National Toxicology Program. Monograph on immunotoxicity associated with exposure to perfluorooctanoic acid (PFOA) and perfluorooctane sulfonate (PFOS). Research Triangle Park, NC: National Toxicology Program; 2016. https://ntp.niehs.nih.gov/ntp/ ohat/pfoa_pfos/pfoa_pfosmonograph_508.pdf.

23. Buekers J, Colles A, Cornelis C, Morrens B, Govarts E, Schoeters G. Socio-economic status and health: evaluation of human biomonitored chemical exposure to per- and polyfluorinated substances across status. Int $\mathrm{J}$ Environ Res Public Health. 2018;15:2818 https://doi.org/10.3390/ijerph15122818.

24. Finch KC, Snook KR, Duke CH, Fu KW, Tse ZTH, Adhikari A, et al. Public health implications of social media use during natural disasters, environmental disasters, and other environmental concerns. Nat Hazards. 2016;83:729-60. https://doi.org/10.1007/ s11069-016-2327-8.

25. English PB, Richardson MJ, Garzón-Galvis C. From crowdsourcing to extreme citizen science: participatory research for environmental health. Annu Rev Public Health. 2018;39:335-50. https://doi.org/10.1146/annurev-publhealth-040617-013702. 